J.D. SMITH

V. SICK

\section{Crank-angle resolved imaging of biacetyl laser-induced fluorescence in an optical internal combustion engine}

Department of Mechanical Engineering, The University of Michigan, 2026 W.E. Lay Automotive Laboratory, 1231 Beal Ave., Ann Arbor, MI 48109-2133, USA

Received: 25 April 2005/Revised version: 12 July 2005 Published online: 24 August 2005 • (C) Springer-Verlag 2005

ABSTRACT The use of a frequency-tripled, diode-pumped Nd:YAG laser in combination with a CMOS camera lens-coupled to a three-stage image intensifier allowed the visualization of the fuel distribution with crank angle resolution for hundreds of consecutive engine cycles. Biacetyl, doped into iso-octane, was excited at rates of $12 \mathrm{kHz}$ with $100 \mathrm{~ns}$ pulses. Pulse energies are high enough to allow single-pulse imaging of the vapor-phase fuel distribution for motored and fired operation in an optical engine. The repetition rate of the setup is adequate to resolve critical steps in the development of the fuel cloud around the spark plug of a direct-injection gasoline engine.

PACS 42.62.Fi; 33.50.Dq; 82.33.Vx

1

\section{Introduction}

Laser-induced fluorescence (LIF) imaging to track the fuel distribution in internal combustion (IC) engines and other combustion devices is one of the most widely used applications of laser-based imaging diagnostics. The importance of the fuel distribution in governing combustion performance has motivated and pushed the development and application of a wide range of LIF-based techniques to assist research and development efforts. Most techniques that attempt to provide quantitative measures of the fuel concentration or equivalence ratio use a surrogate non-fluorescing fuel and a fluorescent tracer [1]. The variations used to date are numerous and are often custom-tailored to a specific measurement task. Usually, high-power pulsed lasers, like excimer lasers, $\mathrm{Nd}$ :YAG lasers with harmonic wavelength conversion, and dye lasers are used as excitation sources in the ultraviolet or near-ultraviolet spectral range. CCD cameras, often equipped with image intensifiers, are well estab- lished as detectors in typical experimental setups. Commonly, the imaging systems have limited acquisition rates of a few frames per second. This frequency is much slower than those of engine related phenomena such as fuel injection, ignition and combustion, which tend to occur on the micro- and millisecond levels. Some improvements have been made to increase frame rates to engine related speeds by using multisensor cameras or cameras with multiexposure capability on a single chip, but these techniques were generally limited to a small number of frames $(\sim 10)$ before having to pause for the camera chip(s) to be read [2-4] and or due to the limited number of pulses emitted by a laser cluster [2].

Film-based systems allowed a higher number of frames to be recorded for Mie scattering experiments at $4 \mathrm{kHz}$ frame rate using a copper vapor laser [5]. The emission wavelength of copper vapor lasers is unfortunately not suitable to excite commonly used fluorescence tracers for fuel distribution studies. More recently, advances in digital camera tech- nology have made it possible to record at $\mathrm{kHz}$-rates (up to $>100 \mathrm{kHz}$ ), and to do so for a significant number of frames. This has allowed for the visualization of naturally occurring combustion intermediates such as the $\mathrm{OH}$ radical $\left(\mathrm{OH}^{*}\right)$ or soot luminosity throughout entire engine cycles [6,7]. A combination of double-pulse PLIF of toluene using two excimer lasers and chemiluminescence imaging of $\mathrm{OH}^{*}$ with a single highspeed camera yielded only two LIF images per engine cycle since the laser repetition rates were limited to one pulse per engine cycle [8]. Even though these sequences could be recorded for several hundred consecutive engine cycles, only two out of seventy images per cycle contain information about the evolution of the fuel distribution, while the remaining 68 only contained signal from $\mathrm{OH}^{*}$. The problem thus remains that a technique must be developed to measure vaporized fuel distribution, in a potentially quantitative fashion, at a high level of spatial resolution and adequate image repetition rate over several engine cycles.

With the advancement of high-power and high-repetition rate Nd:YAG lasers, it is now possible to have an excitation source that operates at the speeds necessary to follow engine phenomena in real-time. These lasers emit naturally at $1064 \mathrm{~nm}$, however it is common to frequency double, triple or quadruple to achieve light at $532 \mathrm{~nm}, 355 \mathrm{~nm}$ or $266 \mathrm{~nm}$, respectively. As the frequency is increased, however, efficiency decreases so it desirable to utilize the highest wavelength possible. Popular fuel tracers such as toluene and acetone both have absorption bands which are easily accessible to the fourth harmonic at 
$266 \mathrm{~nm}$. Typical pulse energies of highrepetition rate $\mathrm{Nd}$ : YAG lasers at $266 \mathrm{~nm}$ are in the $\mu \mathrm{J}$-range, which is too low for PLIF diagnostics in engines. However, at $355 \mathrm{~nm}$, pulse energies close to the $\mathrm{mJ}$-level can be achieved with commercial systems at repetition rates of tens of $\mathrm{kHz}$. Therefore, if a suitable tracer can be used, PLIF imaging at sustained $\mathrm{kHz}$ rates will become feasible.

Biacetyl is one such species with a relatively broad near-UV-Vis absorption band stretching from $340 \mathrm{~nm}$ to $470 \mathrm{~nm}[9,10]$. The use of biacetyl as a tracer for fuel imaging in engines has been described by Baritaud and Heinze [11] using a standard frequencytripled Nd:YAG laser operating at $10 \mathrm{~Hz}$. The photophysics of biacetyl has been studied since the late 1950s, yet is not fully characterized for higher temperature and pressure conditions as present in engines. A recent study reports absorption coefficients and fluorescence quantum yields [12] for engine related conditions. The fluorescence emission band is broad, ranging from $420 \mathrm{~nm}$ to $520 \mathrm{~nm}$ with a peak near $485 \mathrm{~nm}[9,13]$ and there also exists a strong phosphorescence emission band from $475 \mathrm{~nm}$ to $675 \mathrm{~nm}$ [9] that is suppressed at high temperatures $\left(>200^{\circ} \mathrm{C}\right)$ as well as in the presence of oxygen [14]. The substantial separation of fluorescence emission from the excitation at $355 \mathrm{~nm}$ makes it attractive, for it is important to have the ability to suppress scattered laser light both from Mie scattering and from surface reflections in the engine. It should also be noted that the use of biacetyl and excitation at $355 \mathrm{~nm}$ instead of $266 \mathrm{~nm}$ is potentially useful for applications in engines with regular gasoline. Gasoline has very strong absorption bands below $300 \mathrm{~nm}$ [12] and excitation with short wavelengths leads to attenuation effects that are difficult to correct.

This paper presents a method for conducting LIF measurements of vaporized fuel distribution as demonstrated in an IC engine at one crank-angle-degree (CAD) resolution (12000 frames per second for engine speeds of 2000 RPM). This is done through the use of biacetyl as a fuel tracer excited with a frequency tripled diode-pumped Nd:YAG laser at $355 \mathrm{~nm}$. Crank angle resolved image sequences from consecutive engine cycles, or oscillatory combustion processes in gas turbine combustors, are expected to provide insight into causes of random and rare events that lead to combustion instabilities, misfires, or extinction.

\section{Experiment}

The imaging system involves a high-speed CMOS camera (Vision Research Phantom V7.1) which is capable of capturing $4800 \mathrm{fps}$ at full $800 \times$ 600 pixel resolution. With an increase in frame rate, the readable sensor area is decreased. The camera can capture images at up to $150000 \mathrm{fps}$ at a reduced resolution of $32 \times 32$ pixels. The goal of this work was to acquire images for each crank angle degree. When an engine is running at $2000 \mathrm{RPM}$, the frame rate must then be set to 12000 frames per second resulting in a resolution of $386 \times 386$ pixels. At this rate, the system is capable of storing over 38000 frames in a single experimental run. The camera uses a memory gating function which allows for recording only during certain periods of the engine cycle. By utilizing this, the portions of the cycle that are of interest (e.g. injection, vaporization and combustion during the compression and early expansion stroke) can be recorded, while the remainder of the cycle will not be. This allows for many more cycles to be recorded; generally 300 to 400 per experimental run.

To increase signal levels, a lenscoupled image intensifier (LaVision HS-IRO) was used. This three-stage intensifier can be operated at rates up to $100000 \mathrm{fps}$ and shutter times down to 200 ns. Fluorescence signals were focused onto the intensifier with a Nikkon $105 \mathrm{~mm}\left(f_{\#}=1.2\right)$ lens. The transmission of this lens decreases rapidly below $400 \mathrm{~nm}$. In combination with a WG 385 filter (Schott), this helped to efficiently suppress the recording of Mie scattering and scattered laser light from surfaces.

The excitation source was a frequency tripled Nd:YAG laser (Quantronix Hawk) capable of firing up to 50000 times per second. At $12000 \mathrm{~Hz}$, the average power is approximately $5 \mathrm{~W}$, which roughly translates to $0.41 \mathrm{~mJ}$ per pulse. At this repetition rate, the pulse width is approximately $100 \mathrm{~ns}$. The circular beam (approximate $0.6 \mathrm{~mm}$ diameter at outlet) was directed at a $45^{\circ}$ mirror which reflects $355 \mathrm{~nm}$ light while transmitting $532 \mathrm{~nm}$ light (AR-coating on backside). This is necessary to improve the separation of second and third harmonic wavelengths. If the second harmonic light $(532 \mathrm{~nm})$ is allowed in the engine, reflected and scattered light would pass through the filter setup, which then would pollute the collected LIF signal. After the mirror, the light was directed through sheet-forming optics (Rodenstock) to create a light sheet with a cross section of approximately $35 \times 0.5 \mathrm{~mm}$ near the spark plug in the engine (Fig. 1).

The engine is a single cylinder fourstroke direct-injected spark-ignition variant, displacing $0.5 \mathrm{~L}$ (Fig. 2). Optical access for the laser sheet is provided by a quartz window on the surface of the extended length piston (Bowditch type) and a $45^{\circ}$ turning mirror. Signal collection is by way of a full length quartz cylinder liner and quartz windows in the cylinder head. The piston has a dish type feature on the top surface to aid in fuel stratification, so quartz windows are also present on the side of the piston to allow viewing inside this bowl $[8,15]$.

The engine can be motored or fired at speeds between 600 and 2400 RPM, although all tests conducted for this experiment were performed at $2000 \mathrm{RPM}$. A hydraulic dynamometer is used to control engine speed and is accurate

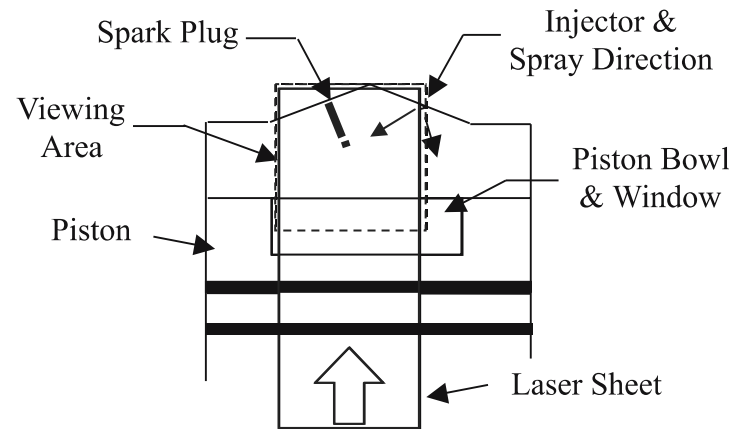

FIGURE 1 Schematic of viewing area within the engine 


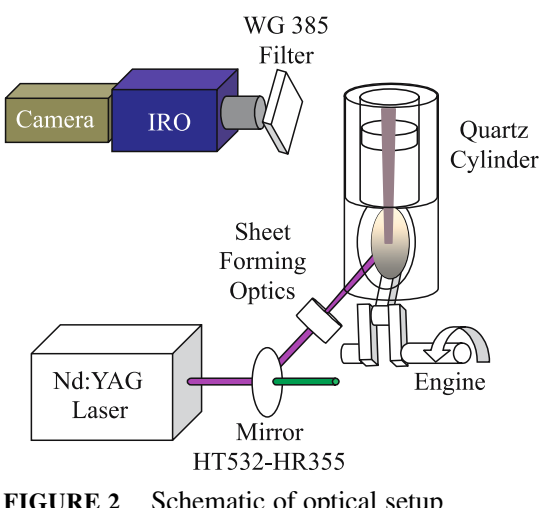

FIGURE 2 Schematic of optical setup

within approximately $+/-20$ RPM. The coolant, oil and intake air are all conditioned to $95^{\circ} \mathrm{C}$ prior to running and intake air is metered through a system of critical orifices. Molecular nitrogen can be added as a diluent when necessary by way of a critical orifice. The fuel (iso-octane with $10 \mathrm{vol} . \%$ biacetyl) is delivered through an 8-hole fuel injector operating at a line pressure of 110 bar, pressurized by nitrogen using a hydraulic accumulator (Tobul). Injection and ignition events are controlled by a setpoint controller (GMR V5.7) synchronized to crankshaft and camshaft encoders. The camera was triggered directly off the crankshaft encoder of the engine to ensure synchronization of the frames to desired crank angle positions. The laser and the intensifier were both triggered using the strobe function from the camera to synchronize them with the electronic shutter of the camera.

The procedure to run all experiments mentioned in this work first involved motoring the engine at 2000 RPM. Once the speed was stable, the fuel injector was turned on and in cases where the engine was fired, ignition was also turned on. When firing, the speed will initially increase significantly as the dynamometer adapts to the power produced by the engine. This makes it necessary to wait approximately 30 seconds for the speed to stabilize, which also allows for the thermal state of the combustion chamber to become more uniform. Then the imaging sequence is initiated and the camera records one image per crank angle during the specified section of each sequential cycle until the on-board memory of the camera is full $(8.2 \mathrm{~GB}$ or approximately 38000 images). The recording portion of the experiment usually takes less than 30 seconds, which minimizes drifts in thermal conditions within the engine.

\section{$3 \quad$ Results}

Data was first acquired with the engine motored. Fuel was injected every other cycle, which allowed for images containing only background noise to be recorded for post processing purposes. During these initial runs, the intensifier gain setting was optimized to provide the most LIF signal while maintaining an acceptable level of background noise. The optimum setting was determined to be $7.9 / 10$, corresponding to a sensitivity of approximately 900 counts per photoelectron.

Once the data was acquired, it was transferred via 16-bit Tagged Image Format into an image processing software (LaVision DaVis 7) for post processing. Since the camera operates with 12-bit dynamic range, the intensity scale is linearly increased from 0-4096 to 065536 in this conversion step. The first processing step was to smooth the images to reduce shot noise influence. For this purpose, a 5 pixel sliding average filter was applied. This results in a spatial resolution of approximately $0.3 \mathrm{~mm}$ which is still less than the $0.5 \mathrm{~mm}$ thickness of the lightsheet. The background images were averaged over 30 cycles, and then were subtracted from the images at each respective crank angle. Following smoothing and background subtraction, a mask was applied to suppress reflections from the cylinder head and an overlay was applied to clarify the position of significant features within the combustion chamber. The final step involves dividing the images by an averaged image of a well mixed distribution of fuel and air. This step removes any influences of non-homogeneities in the laser light sheet as well as the detection path. The well mixed condition was met by injecting fuel early in the intake stroke ( $\left.345^{\circ} \mathrm{BTDC}\right)$. These images were then processed similarly to the others, except a 7-pixel sliding average filter was applied.

A sample of the results from a motored engine run without ignition can be seen in Fig. 3, which displays a time sweep spanning 20 crank angle degrees (CAD). The sweep begins at $33^{\circ}$ before top-dead-center (BTDC). Previous Mie scattering studies completed on this en- gine confirm that all but a few liquid droplets are vaporized by this point, so we can be confident that the LIF signal displayed is mainly due to fuel vapor and not from fuel droplets [16]. Note that Mie scattering is efficiently suppressed by the optical filter and the lens in use. A bright area is present on the left corner of the spark plug electrode tip through $21^{\circ}$ BTDC. The gradual decrease in signal of this area raises the possibility that this is liquid fuel evaporating from the surface of the spark plug. It can be noted that, following impact with the spark plug, the fuel cloud branches off in two directions. The section that follows the original trajectory of the spray (straight through the spark gap) tends to stop fairly quickly and actually moves back towards the center of the cylinder. This is likely due to a significant squish flow generated by the rising piston, which tends to force air from the outer edges of the cylinder towards the piston bowl. The second section of the fuel cloud gets directed downward into the piston bowl. This section tends to be initially stationary directly beneath the spark plug, then gradually moves towards the right side of the piston bowl. It is worth noting that previous studies [8] completed in this engine focusing on $\mathrm{OH}^{*}$ production have indicated that the flame consistently moves in a similar fashion as fuel cloud has been found in this study to move. This is also in agreement with flow field measurements [16] that show a strong tumble flow in the direction that is observed through the motion of the fuel plume. Finally, it has to be noted that the absorption coefficient of biacetyl at $355 \mathrm{~nm}$ is independent of temperature over the crank angle range discussed here $(550 \mathrm{~K} \sim 690 \mathrm{~K})$ with a statistical uncertainty of $20 \%$ and the fluorescence quantum yield remains unchanged within $10 \%$ for increasing temperature and pressure [12]. Thus, for this paper the signals were not corrected for temperature and pressure effects. Due to the decrease of the in-cylinder volume by almost a factor of two from $35^{\circ}$ BTDC to $14^{\circ}$ BTDC the number density increases by that factor which is reflected in the images shown in Fig. 3.

From cycle to cycle, there is significant variation in the measured LIF intensity at locations around the viewing area. However, a general trend is for the fuel cloud to move in a counter- 


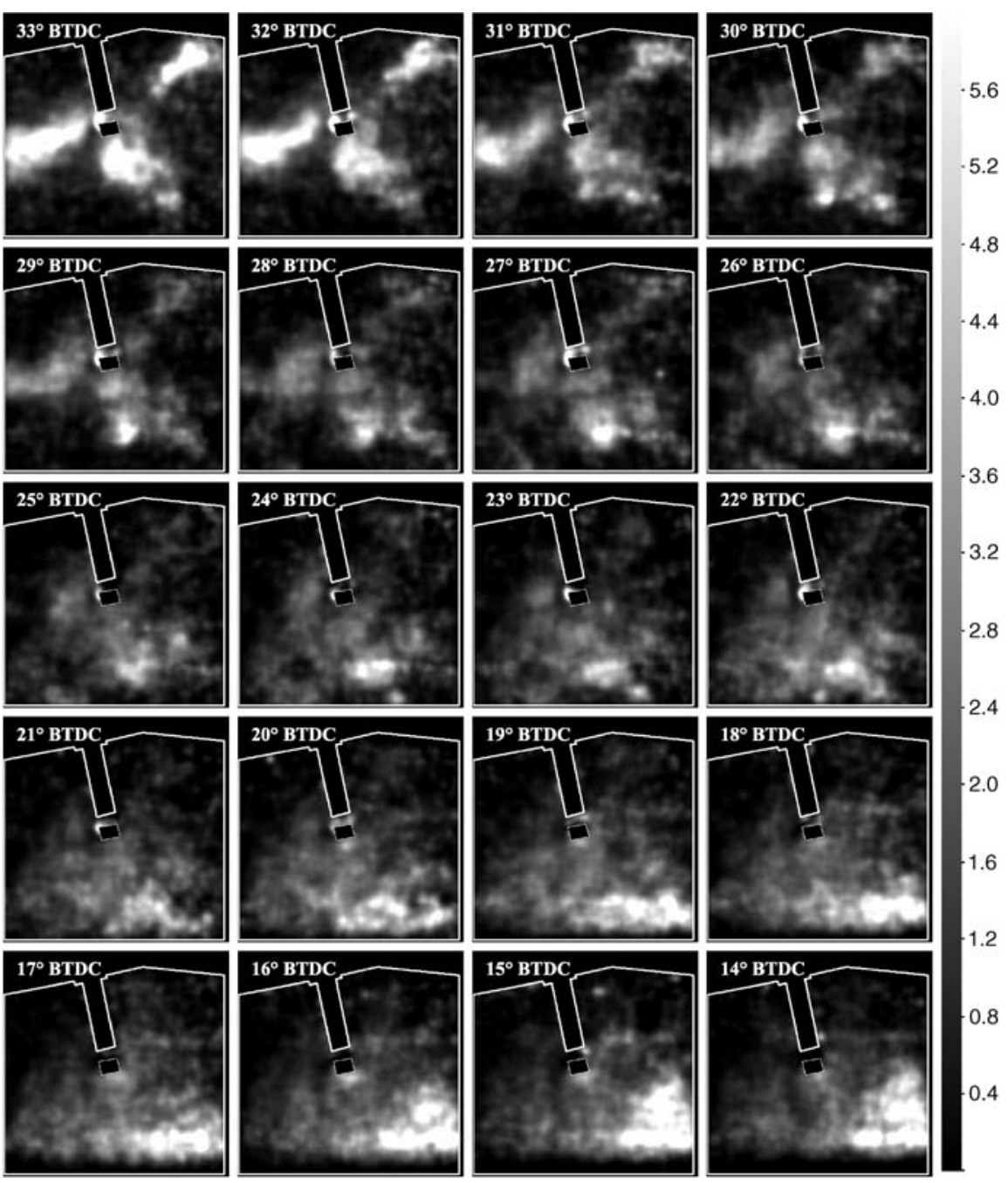

FIGURE 3 Time sequence of biacetyl LIF images from the same engine cycle under motored conditions. The images were normalized to the light sheet intensity distribution and are shown in an arbitrary intensity scaling. The fuel jet is split into two jets upon impact on the spark plug electrodes

clockwise motion. It is highly encouraging that not just this bulk behavior can be observed, but also the cycle-to-cycle variations in details of the fuel distribution, which likely have a significant effect on combustion stability.

Given the low signal to noise ratio and the limited dynamic range of the images presented here, it is crucial to address the significance of the information obtained. To this end, LIF images of toluene that were acquired upon excitation with $\mathrm{KrF}$ excimer lasers in the same engine under the same operating conditions were analyzed. These images have a substantially higher dynamic range and signal to noise ratio [8]. An assessment of phase-averaged images (35 images) and their rms variation revealed that the ensemble variations were on the order of $25 \%-30 \%$ for images taken early during the injec- tion process. Because of the high image quality, these results describe the level of cyclic fluctuations of the fuel distribution. For the biacetyl images obtained in the present study, i.e. at $35^{\circ}$ BTDC when spatial fluctuations are expected to be lowest, fluctuations on the order of $25 \%$ were found as well, confirming that the image quality is high enough to derive meaningful information. At later crank angles, rms variations increased to about $45 \%$ due to substantially increased spatial variations in fuel distribution in different cycles as can qualitatively be observed from raw images. The spatial structures that are observed in individual cycles, despite the relatively low $\mathrm{S} / \mathrm{N}$ ratio and $\mathrm{dy}-$ namic range for the present setup, are significant in the context of the measured rms values and therefore provide useful information.
During combustion, it can be expected that the biacetyl fluorescence is overlapped by chemiluminescence from e.g. $\mathrm{CH}, \mathrm{C}_{2}$ and soot luminosity. Usually, detection of chemiluminescence can be suppressed in favor of the LIF signal by narrowing the integration (gate) time of the intensifier to match the laser pulse duration plus fluorescence decay time. Here this amounts to approximately $100 \mathrm{~ns}$, well matched to the minimum gate time of $200 \mathrm{~ns}$. Due to the high stratification of the fuel, areas of rich combustion tend to produce very intense signals, likely from hot soot. There is a significant level of signal from the flame introduced in the LIF images. This is of no consequence to the LIF images until approximately $25^{\circ}$ BTDC when the flame begins to occupy a significant portion of the viewing area. Figure 4 shows a comparison of images for fired engine operation with those from the motored case. These three crank angles were chosen to show distinct features. A significant amount of liquid fuel is present at $35^{\circ}$ BTDC which leads to the very strong signals in the shape of the original spray plume. The presence of liquid fuel at $35^{\circ} \mathrm{BTDC}$ is confirmed by previous Mie scattering studies as is the discontinuous structure of the spray plume. At $30^{\circ}$ BTDC the signal is largely due to vaporized fuel and the arc can be noted in the gap of the spark plug. By this point, only $3.5^{\circ}$ have passed (approximately $300 \mu \mathrm{s}$ ) since the breakdown of the spark, so very little burning has occurred and the LIF signal is similar to that of the motored case. At $25^{\circ} \mathrm{BTDC}$, combustion signals begin to become apparent when compared with the motored case. Past this point, the viewing area is rapidly engulfed in chemiluminescence signal. One focus area of future work will be attempting to mitigate this chemiluminescence possibly using bandpass filters or colored glass filters.

As mentioned earlier, biacetyl produces a strong phosphorescence signal at lower temperatures and in the absence of oxygen. This signal is generally long lasting, with lifetimes on the order of a millisecond. This, combined with the high level of temperature dependence, makes the phosphorescence signal detrimental to any attempts at making quantitative measurements in 


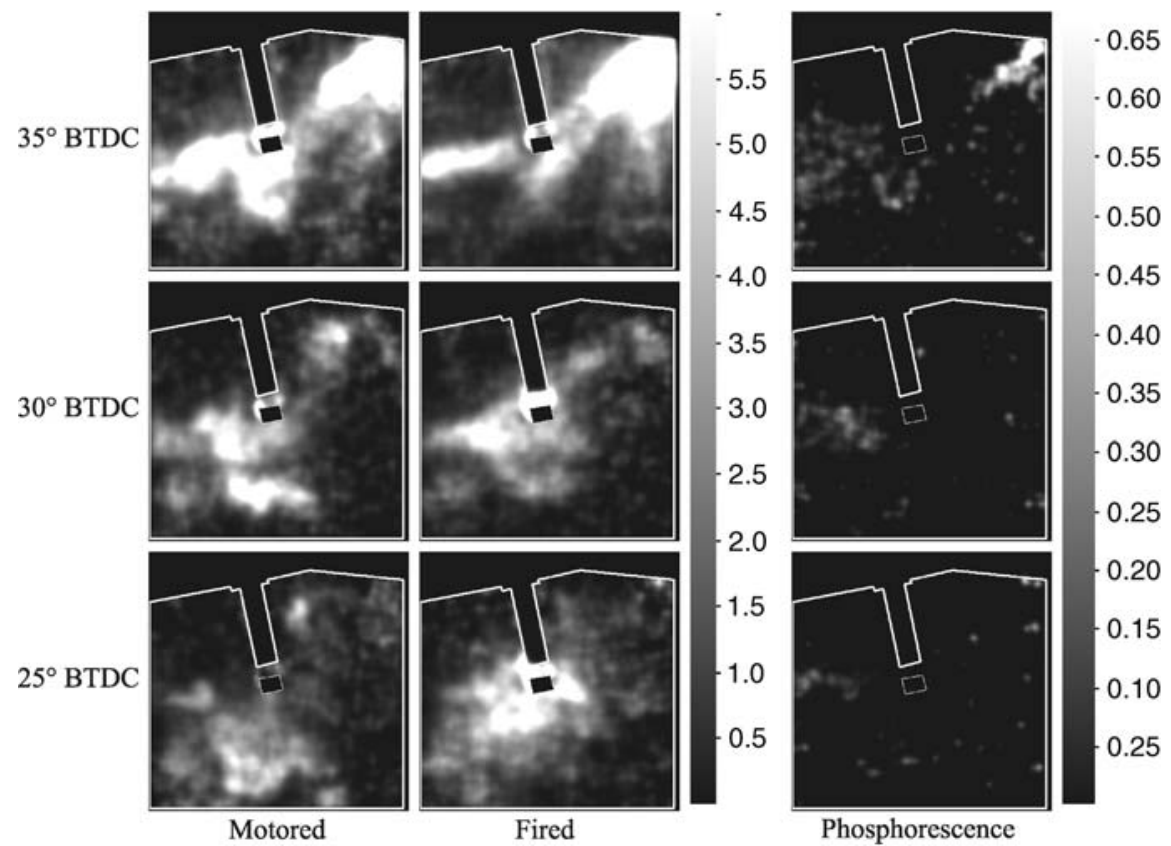

FIGURE 4 Comparison of signal from two engine operating conditions and the signal from phosphorescence at three engine positions. Motored and fired conditions are displayed on the same intensity scale, whereas phosphorescence is not. Intensity is displayed in arbitrary units

the present context and detection should be suppressed.

To determine if phosphorescence was indeed contributing significantly to our signal, an experiment was conducted which would yield only signal from phosphorescence. To do this, an OG 550 filter was used instead of the WG 385. The fluorescence emission band quickly tapers off beyond approximately $530 \mathrm{~nm}$ and the measured transmission of the filter at this point is about 1\% (using an Ocean Optics USB 2000 spectrometer), therefore we can be confident that fluorescence will not contribute significantly to the signal. The phosphorescence signal, however, has strong emission peaks at 512, 560 and $610 \mathrm{~nm}$ [9], the latter two of which pass through the filter without much attenuation.

The motored experiment was repeated with the OG 550 filter in place, and the images were post processed consistent with the other cases. The images from three engine positions are presented in Fig. 4, along with the fluorescence from the motored and fired cases, as discussed earlier. The phosphorescence images are presented at a higher resolution to enhance the structure of the phosphorescence signal distribution. At $35^{\circ}$ BTDC, the phosphorescence signal is greatest, which is what would be expected. The relatively cold fuel and evaporative cooling may locally lower the temperature in areas of heavy liquid concentration to levels at which phosphorescence may become active. In the core of the liquid spray jet, it is also expected that oxygen concentrations are minimal. At the two later times, the phosphorescence is only visible in sporadic areas, in intensities very close to the noise limit. Averages taken in three areas of the viewing area for comparison purposes between phosphorescence images and fluorescence images consistently show a one, and sometimes two, order of magnitude difference between the signals. From these results, we can indeed be confident in believing that phosphorescence does not contribute significantly to our signal.

\section{Conclusions}

Single-pulse fuel imaging capability at frame rates of $12 \mathrm{kHz}$ for hundreds of consecutive cycles of an optical engine was demonstrated. A tripled diode-pumped Nd:YAG laser produced pulses of $100 \mathrm{~ns}$ duration and approximately $0.41 \mathrm{~mJ}$ energy that were used to excite biacetyl. Biacetyl was added to iso-octane fuel as a fluorescence tracer. A CMOS camera, lens-coupled with a three-stage image intensifier, was used to sensitively detect the fluorescence signals of the fuel/tracer mixture in the vicinity of the spark plug of a spark-ignition direct-injection engine. Phosphorescence signals are mostly insignificant except at very early stage of the fuel injection process where the fuel temperature and oxygen level within the fuel cloud are low. The impingement and breakup of a fuel jet at the spark plug electrodes could be followed with crank angle resolution. The subsequent fuel stratification within the piston bowl is consistent with the propagation of the flame as observed in an earlier chemiluminescence imaging study. Quantification of biacetyl LIF signals under combined higher temperature and pressure conditions can be pursued in the future using recent measurements of fluorescence quantum yields for those conditions. Even though the signal to noise ratio is substantially below that for typical LIF imaging experiments with high-power UV lasers, the technique described here does provide significant information about in-cylinder fuel distributions and their evolution at unprecedented sustained frames rates.

ACKNOWLEDGEMENTS This work was sponsored by General Motors Corporation through the General Motors-University of Michigan Collaborative Research Laboratory on Engine Systems Research. JDS is grateful to the College of Engineering for a Graduate Student Instructor appointment. The authors are also indebted to James F. Driscoll and Steven L. Ceccio at The University of Michigan for the loan of the Nd:YAG laser.

\section{REFERENCES}

1 C. Schulz, V. Sick: Prog. Energy Combust. Sci. 31, 75 (2005)

2 J. Hult, M. Richter, J. Nygren, M. Aldén, A. Hultqvist, M. Christensen, B. Johansson: Appl. Opt. 41, 5002 (2002)

3 W. Reckers, H. Schwab, C. Weiten, B. Befrui, R. Kneer: 5. Internationales Symposium für Verbrennungsdiagnostik, Baden-Baden, Germany 2002

4 R.B. Miles: In: Applied Combustion Diagnostics, K. Kohse-Höinghaus and J.B. Jeffries (Eds.) (Taylor and Francis, New York 2002)

5 M.C. Drake, T.D. Fansler, D.T. French: SAE Paper 952454 (1995)

6 B.D. Stojkovic, T.D. Fansler, M.C. Drake, V. Sick: Proc. Combust. Inst. 30, 2657 (2005)

7 M.C. Drake, T.D. Fansler, A. Lippert: Proc. Comb. Inst. 30, 2683 (2005)

8 J.D. Smith, V. Sick: Appl. Opt. (2005), accepted for publication

9 A. Lozano, B. Yip, R.K. Hanson: Exp. Fluids 13, 369 (1992) 
10 H. Hu, M.M. Koochesfahani: Exp. Fluids 33, $202(2002)$

11 T. Baritaud, T. Heinze: SAE Paper 922355 (1992)

12 N. Wermuth, V. Sick: SAE Paper 2005-01-
2090 (2005)

13 W. Lawrenz, J. Köhler, F. Meier, W. Stolz, R. Wirth, W.H. Bloss: SAE Paper 922320 (1992)

14 J.B. Liu, Q. Pan, C.S. Liu, J.R. Shi: Exp. Fluids 6, 505 (1988)
15 U. Fissenewert, V. Sick, H. Pucher: SAE Paper 2005-01-2089 (2005).

16 C.M. Fajardo, J.D. Smith, V. Sick: ICOLAD II, accepted, London, UK, 2005 (Institute of Physics) 